\title{
On closed-form solutions to a class of ordinary differential equations
}

\author{
Dhrushil Badani
}

Email: invadeyourdreams@gmail.com

\begin{abstract}
Copyright (C)2014 Dhrushil Badani. This is an open access article distributed under the Creative Commons Attribution License,
\end{abstract} which permits unrestricted use, distribution, and reproduction in any medium, provided the original work is properly cited.

\begin{abstract}
In this paper,closed-form solutions in terms of integral(s) to any second and third order linear non-homogeneous constant-coefficient ordinary differential equations as well as the second order non-homogeneous Cauchy-Euler equation, are derived. The method is generalized for $n^{\text {th }}$ order linear non-homogeneous constant-coefficient ordinary differential equations. A similar method is used to derive closed-form solutions to second order linear non-homogeneous non- constant coefficient ordinary differential equations. We then demonstrate an application of the method to recurrence relations of the second order.
\end{abstract}

Keywords: Closed-form, Ordinary Differential Equations, Recurrence Relations

\section{Introduction}

Existing methods in literature to solve non-homogeneous second order ordinary differential equations involve finding the solution to the associated homogeneous differential equation first and involve guessing of solutions [1, 2, 3]. In contrast, in this paper, we derive the solution for any second order linear non-homogeneous constant-coefficient ordinary differential equation, in terms of certain integral(s), without guessing or finding the solution to the associated homogeneous differential equation or using initial conditions or having one solution to the equation before hand. We also use the same method to derive the solution for second order non-homogeneous Cauchy-Euler equations, in terms of certain integral(s). The solution for any third order linear non-homogeneous constant-coefficient ordinary differential equation, in terms of certain integral(s) is then derived, using the same method, after which there is a generalization for any $n^{\text {th }}$ order linear non-homogeneous constant-coefficient ordinary differential equation. We then propose a method for the case when the coefficents are non-constant, by linking the second-order ordinary differential equation with an associated Riccati differential equation. Once any one solution to the Riccati differential equation is known, we use it in an integral obtain the closed-form solution to the second-order differential equation. We also list closed-form solutions to certain types of equations of this form to further illustrate the method. Lastly, the method is applied to derive closed-form solutions to second order recurrence relations, the discrete counterparts of differential equations.

Note: Wherever the need be (especially when the algebraic equations yield complex roots), we must use Euler's formula viz., $e^{i x}=\cos (x)+\iota \sin (x)$. 


\section{The second order linear non-homogeneous constant-coefficient dif- ferential equation}

In this section, we consider the differential equation of the form:

$$
y^{\prime \prime}+a_{1} y^{\prime}+a_{2} y=f(x)
$$

where $a_{1}$ and $a_{2}$ are constants.

Theorem 2.1. For the differential equation,

$$
y^{\prime \prime}+a_{1} y^{\prime}+a_{2} y=f(x),
$$

the solution is:

$$
y(x)=e^{a x} \int e^{(b-a) x}\left(\int f(x) e^{-b x} \mathrm{~d} x\right) \mathrm{d} x
$$

where, $a$ and $b$ are roots of the quadratic equation:

$$
t^{2}+a_{1} t+a_{2}=0
$$

Proof. Since $a$ and $b$ are roots of the quadratic equation $t^{2}+a_{1} t+a_{2}=0$,

$$
\begin{gathered}
a_{1}=-a-b \\
a_{2}=a b
\end{gathered}
$$

Substituting $a_{1}$ and $a_{2}$ in the differential equation, we have:

$$
y^{\prime \prime}-a y^{\prime}-b y^{\prime}+a b y=f(x)
$$

On re-arranging the terms, we have,

$$
y^{\prime \prime}-a y^{\prime}-b\left(y^{\prime}-a y\right)=f(x)
$$

Let $y^{\prime}-a y=z(x)$. Thus,

$$
y^{\prime \prime}-a y^{\prime}=z^{\prime}(x)=\frac{\mathrm{d} z}{\mathrm{~d} x}
$$

On substituting $z$ and $z^{\prime}$ into the equation, we have:

$$
z^{\prime}-b z=f(x)
$$

Multiply both sides by $e^{-b x}$ ['Integrating factor'],

$$
e^{-b x} z^{\prime}-b z e^{-b x}=f(x) e^{-b x}
$$

The left hand side of this equation is nothing but the derivative of $z e^{-b x}$ with respect to $x$.

$$
\mathrm{d}\left(z e^{-b x}\right)=f(x) e^{-b x} \mathrm{~d} x
$$

Integrate both sides of the equation:

$$
\begin{aligned}
& z e^{-b x}=\int f(x) e^{-b x} \mathrm{~d} x \\
& z=e^{b x} \int f(x) e^{-b x} \mathrm{~d} x
\end{aligned}
$$

Since $z=y^{\prime}-a y$,

$$
y^{\prime}-a y=e^{b x} \int f(x) e^{-b x} \mathrm{~d} x
$$

Multiply both sides by $e^{-a x}$ ['Integrating factor'],

$$
e^{-a x} y^{\prime}-a y e^{-a x}=e^{(b-a) x} \int f(x) e^{-b x} \mathrm{~d} x
$$


The left hand side of this equation is nothing but the derivative of $y e^{-a x}$ with respect to $x$.

$$
\mathrm{d}\left(y e^{-a x}\right)=e^{(b-a) x}\left(\int f(x) e^{-b x} \mathrm{~d} x\right) \mathrm{d} x
$$

Integrate both sides of the equation:

$$
y e^{-a x}=\int e^{(b-a) x}\left(\int f(x) e^{-b x} \mathrm{~d} x\right) \mathrm{d} x
$$

Thus,

$$
y=e^{a x} \int e^{(b-a) x}\left(\int f(x) e^{-b x} \mathrm{~d} x\right) \mathrm{d} x
$$

which ends the proof.

\subsection{The case of the homogeneous differential equation}

In this sub-section, we consider the known result of the special case when $f(x)=0$, i.e. when the differential equation is homogeneous:

$$
y^{\prime \prime}+a_{1} y^{\prime}+a_{2} y=0
$$

The solution in this case would be:

$$
\begin{gathered}
y=e^{a x} \int e^{(b-a) x}\left(\int f(x) e^{-b x} \mathrm{~d} x\right) \mathrm{d} x \\
y=e^{a x} \int e^{(b-a) x}\left(\int 0 \mathrm{~d} x\right) \mathrm{d} x \\
y=e^{a x} \int c e^{(b-a) x} \mathrm{~d} x \\
y=e^{a x}\left(\frac{c}{b-a} e^{(b-a) x}+c_{2}\right)
\end{gathered}
$$

Let $\frac{c}{b-a}=c_{1}$. Then,

$$
\begin{gathered}
y=e^{a x}\left(c_{1} e^{(b-a) x}+c_{2}\right) \\
y=c_{1} e^{b x}+c_{2} e^{a x}
\end{gathered}
$$

where, $c_{1}$ and $c_{2}$ are constants (of integration), and $a$ and $b$ are as defined above.

Thus, $y=c_{1} e^{b x}+c_{2} e^{a x}$ is the solution to the second order linear constant-coefficient homogeneous differential equation which validates Theorem 2.1 when instantiated for the homogeneous equation as a special case and is consistent with the known result.

Corollary 2.1. The solution to the second-order linear non-homogeneous constant-coefficient differential equation always contains terms representing the solution to the associated homogeneous differential equation.

Proof. From Theorem 2.1,

$$
y=e^{a x} \int e^{(b-a) x}\left(\int f(x) e^{-b x} \mathrm{~d} x\right) \mathrm{d} x
$$

is the solution to the second-order linear non-homogeneous constant-coefficient differential equation, where $a$ and $b$ are as defined above.

Set

$$
\int f(x) e^{-b x} \mathrm{~d} x=g(x)+c
$$

where $c$ is the constant of integration. Thus,

$$
y=e^{a x} \int e^{(b-a) x}(g(x)+c) \mathrm{d} x
$$




$$
y=e^{a x}\left(\int e^{(b-a) x} g(x) \mathrm{d} x+c_{1} e^{(b-a) x}+c_{2}\right)
$$

where,

$$
c_{1}=\frac{c}{b-a}
$$

and $c_{2}$ is the constant of integration. Thus,

$$
y=e^{a x}\left(\int e^{(b-a) x} g(x) \mathrm{d} x\right)+c_{1} e^{b x}+c_{2} e^{a x}
$$

Thus, the last two terms of this expression represent the terms from the solution to the associated homogeneous differential equation as seen above.

\section{The second order non-homogeneous cauchy-euler equation}

The second-order non-homogeneous Cauchy-Euler equation is of the form:

$$
x^{2} y^{\prime \prime}+k x y^{\prime}+m y=f(x)
$$

Consider a more general equation of the form:

$$
\left(a_{1} x+a_{2}\right)^{2} y^{\prime \prime}+k\left(a_{1} x+a_{2}\right) y^{\prime}+m y=f(x)
$$

where, $a_{1}, a_{2}, k$ and $m$ are constants.

Theorem 3.1. For the differential equation

$$
\left(a_{1} x+a_{2}\right)^{2} y^{\prime \prime}+k\left(a_{1} x+a_{2}\right) y^{\prime}+m y=f(x),
$$

the solution is of the form

$$
y(x)=\left(a_{1} x+a_{2}\right)^{a} \int\left(a_{1} x+a_{2}\right)^{b-a-1}\left(\int \frac{f(x)}{a_{1} x+a_{2}} \mathrm{~d} x\right) \mathrm{d} x
$$

where $a$ and $b$ are roots of the quadratic equation:

$$
t^{2}+\frac{k-a_{1}}{a_{1}} t+\frac{m}{a_{1}^{2}}=0
$$

Proof. Set

$$
\begin{gathered}
\ln \left(a_{1} x+a_{2}\right)=t, \\
y=g(t)
\end{gathered}
$$

Thus,

$$
\begin{gathered}
x=\frac{e^{t}-a_{2}}{a_{1}} \\
y^{\prime}=\frac{\mathrm{d}(g(t))}{\mathrm{d} x}=\frac{a_{1}}{a_{1} x+a_{2}} g^{\prime}(t) \\
y^{\prime \prime}=\frac{\mathrm{d}^{2}(g(t))}{\mathrm{d} x^{2}}=\frac{a_{1}^{2}}{\left(a_{1} x+a_{2}\right)^{2}}\left(g^{\prime \prime}(t)-g^{\prime}(t)\right)
\end{gathered}
$$

Substitute $x, y, y^{\prime}$ and $y^{\prime \prime}$ in the differential equation:

$$
\left(a_{1} x+a_{2}\right)^{2} y^{\prime \prime}+k\left(a_{1} x+a_{2}\right) y^{\prime}+m y=f(x)
$$

On simplifying, we have:

$$
a_{1}^{2}\left(g^{\prime \prime}(t)-g^{\prime}(t)\right)+k a_{1} g^{\prime}(t)+m g(t)=f\left(\frac{e^{t}-a_{2}}{a_{1}}\right)
$$




$$
a_{1}^{2} g^{\prime \prime}(t)+\left(k a_{1}-a_{1}^{2}\right) g^{\prime}(t)+m g(t)=f\left(\frac{e^{t}-a_{2}}{a_{1}}\right)
$$

Dividing by $a_{1}^{2}$,

$$
g^{\prime \prime}(t)+\frac{k-a_{1}}{a_{1}} g^{\prime}(t)+\frac{m}{a_{1}^{2}} g(t)=\frac{1}{a_{1}^{2}} f\left(\frac{e^{t}-a_{2}}{a_{1}}\right)
$$

Now, this is a second order linear constant-coefficient non-homogeneous differential equation in $g(t)$.

From Theorem 2.1, the solution to this equation is:

$$
g(t)=\frac{e^{a t}}{a_{1}^{2}} \int e^{(b-a) t}\left(\int f\left(\frac{e^{t}-a_{2}}{a_{1}}\right) d t\right) d t
$$

where $a$ and $b$ are roots of the quadratic equation:

$$
t^{2}+\frac{k-a_{1}}{a_{1}} t+\frac{m}{a_{1}^{2}}=0
$$

Since,

$$
t=\ln \left(a_{1} x+a_{2}\right)
$$

and

$$
y=g(t)
$$

it follows that,

$$
\begin{aligned}
e^{t} & =\left(a_{1} x+a_{2}\right), \\
e^{(b-a) t} & =\left(a_{1} x+a_{2}\right)^{(b-a)} \\
\mathrm{d} t & =\frac{a_{1}}{a_{1} x+a_{2}} \mathrm{~d} x
\end{aligned}
$$

Thus,

$$
y(x)=\left(a_{1} x+a_{2}\right)^{a} \int\left(a_{1} x+a_{2}\right)^{b-a-1}\left(\int \frac{f(x)}{a_{1} x+a_{2}} \mathrm{~d} x\right) \mathrm{d} x
$$

which completes the proof.

\section{The third order linear non-homogeneous constant-coefficient differ- ential equation}

In this section, we consider the differential equation of the form:

$$
y^{\prime \prime \prime}+a_{1} y^{\prime \prime}+a_{2} y^{\prime}+a_{3} y=f(x)
$$

Theorem 4.1. For the differential equation

$$
y^{\prime \prime \prime}+a_{1} y^{\prime \prime}+a_{2} y^{\prime}+a_{3} y=f(x)
$$

the solution is of the form:

$$
y(x)=e^{c_{1} x} \int e^{\left(c_{2}-c_{1}\right) x}\left(\int e^{-\left(b_{3}+c_{2}\right) x}\left(\int e^{b_{3} x} f(x) \mathrm{d} x\right) \mathrm{d} x\right) \mathrm{d} x
$$

where $b_{1}, b_{2}$ and $b_{3}$ are constants satisfying the following algebraic equations:

$$
\begin{gathered}
b_{1}+b_{3}=a_{1} \\
b_{2}+b_{1} b_{3}=a_{2}
\end{gathered}
$$




$$
b_{2} b_{3}=a_{3}
$$

and,

$c_{1}$ and $c_{2}$ are the roots of the quadratic equation:

$$
t^{2}+b_{1} t+b_{2}=0
$$

Proof. Set

$$
\begin{gathered}
b_{1}+b_{3}=a_{1} \\
b_{2}+b_{1} b_{3}=a_{2} \\
b_{2} b_{3}=a_{3}
\end{gathered}
$$

Thus, the differential equation becomes

$$
y^{\prime \prime \prime}+\left(b_{1}+b_{3}\right) y^{\prime \prime}+\left(b_{2}+b_{1} b_{3}\right) y^{\prime}+\left(b_{2} b_{3}\right) y=f(x)
$$

Regrouping the terms, we have,

$$
\frac{\mathrm{d}}{\mathrm{d} x}\left(y^{\prime \prime}+b_{1} y^{\prime}+b_{2} y\right)+b_{3}\left(y^{\prime \prime}+b_{1} y^{\prime}+b_{2} y\right)=f(x)
$$

Set

$$
y^{\prime \prime}+b_{1} y^{\prime}+b_{2} y=z(x)
$$

Thus, the differential equation becomes

$$
z^{\prime}+b_{3} z=f(x)
$$

Multiply both sides of the equation by $e^{b_{3} x}$ ['Integrating factor'],

$$
z^{\prime} e^{b_{3} x}+z b_{3} e^{b_{3} x}=f(x)
$$

The left hand side of this equation is the derivative of $z e^{b_{3} x}$

$$
\mathrm{d}\left(z e^{b_{3} x}\right)=e^{b_{3} x} f(x) \mathrm{d} x
$$

Integrate both sides of the equation:

$$
\begin{gathered}
z e^{b_{3} x}=\int e^{b_{3} x} f(x) \mathrm{d} x \\
z=e^{-b_{3} x} \int e^{b_{3} x} f(x) \mathrm{d} x
\end{gathered}
$$

Since

$$
z=y^{\prime \prime}+b_{1} y^{\prime}+b_{2} y
$$

The differential equation becomes

$$
y^{\prime \prime}+b_{1} y^{\prime}+b_{2} y=e^{-b_{3} x} \int e^{b_{3} x} f(x) \mathrm{d} x
$$

Now, this is a second order linear constant-coefficient non-homogeneous differential equation in $y$.

From Theorem 2.1, the solution to this equation is:

$$
y(x)=e^{c_{1} x} \int e^{\left(c_{2}-c_{1}\right) x}\left(\int e^{-\left(b_{3}+c_{2}\right) x}\left(\int e^{b_{3} x} f(x) \mathrm{d} x\right) \mathrm{d} x\right) \mathrm{d} x
$$

where $c_{1}$ and $c_{2}$ are the roots of the quadratic equation:

$$
t^{2}+b_{1} t+b_{2}=0
$$

This completes the proof. 


\section{Generalization for the Nth order linear non-homogeneous constant- coefficient differential equation}

In this section, a method to find the solution to any $n^{\text {th }}$ order linear constant-coefficient non-homogeneous differential equations is presented. Consider the $n^{\text {th }}$ order differential equation of the form:

$$
\begin{gathered}
y^{(n)}+a_{1} y^{(n-1)}+a_{2} y^{(n-2)}+\cdots \cdots+a_{n} y=f(x) \\
y^{(n)}+\sum_{i=1}^{n} a_{i} y^{(n-i)}=f(x)
\end{gathered}
$$

where, $y^{(r)}$ denotes the $r^{t h}$ order derivative of $y$ with respect to $x$.

Now,set

$$
\begin{gathered}
a_{2}=b_{2}+b_{1} b_{n} \\
a_{3}=b_{3}+b_{2} b_{n} \\
\vdots \\
\vdots \\
a_{n-1}=b_{n-1}+b_{n-2} b_{n}
\end{gathered}
$$

and,

$$
\begin{aligned}
& a_{1}=b_{1}+b_{n} \\
& a_{n}=b_{n-1} b_{n}
\end{aligned}
$$

where, $b_{1}, b_{2} \ldots b_{n}$ are unknowns to be solved for.

Thus, the differential equation becomes:

$$
y^{(n)}+\left(b_{1}+b_{n}\right) y^{(n-1)}+\left(b_{2}+b_{1} b_{n}\right) y^{(n-2)}+\cdots \cdots\left(b_{n-1}+b_{n-2} b_{n}\right) y^{\prime}+\left(b_{n-1} b_{n}\right) y=f(x)
$$

Regrouping the terms, we have,

$$
\begin{gathered}
\frac{\mathrm{d}}{\mathrm{d} x}\left(y^{(n-1)}+b_{1} y^{(n-2)} \cdots b_{n-1} y\right)+b_{n}\left(y^{(n-1)}+b_{1} y^{(n-2)}+b_{2} y^{(n-3)} \cdots b_{n-1} y\right)=f(x) \\
\frac{\mathrm{d}}{\mathrm{d} x}\left(y^{(n-1)}+\sum_{i=1}^{n-1} b_{i} y^{(n-i-1)}\right)+b_{n}\left(y^{(n-1)}+\sum_{i=1}^{n-1} b_{i} y^{(n-i-1)}\right)=f(x)
\end{gathered}
$$

Set

$$
y^{(n-1)}+\sum_{i=1}^{n-1} b_{i} y^{(n-i-1)}=z(x)
$$

Thus, the differential equation can be written as:

$$
z^{\prime}+b_{n} z=f(x)
$$

Multiply both sides of the equation by $e^{b_{n} x}$ : [" 'Integrating Factor']

$$
z^{\prime} e^{b_{n} x}+b_{n} e^{b_{n} x} z=e^{b_{n} x} f(x)
$$

The left hand side of the equation is the derivative of $z e^{b_{n} x}$ with respect to $x$.

$$
\mathrm{d}\left(z e^{b_{n} x}\right)=e^{b_{n} x} f(x) \mathrm{d} x
$$

Integrate both sides of the equation:

$$
z e^{b_{n} x}=\int e^{b_{n} x} f(x) \mathrm{d} x
$$




$$
z=e^{-b_{n} x} \int e^{b_{n} x} f(x) \mathrm{d} x
$$

Since,

$$
z=y^{(n-1)}+\sum_{i=1}^{n-1} b_{i} y^{(n-i-1)}
$$

The differential equation becomes

$$
y^{(n-1)}+\sum_{i=1}^{n-1} b_{i} y^{(n-i-1)}=e^{-b_{n} x} \int e^{b_{n} x} f(x) \mathrm{d} x
$$

This equation again is a linear constant-coefficient non-homogeneous differential equation of order $(n-1)$. The method presented above can be applied repetitively by setting up equations in the pattern shown above, until a solution is attained in terms of the unknowns found during the repetitions. The motivation of the method is to simply enable effective regrouping of terms, so as to form a first order linear differential equation, solvable by simply multiplying both sides of it by the appropriate 'Integrating Factor.'

\section{The second order linear non-homogeneous non-constant coefficient differential equation}

In this section, we consider the differential equation of the form:

$$
y^{\prime \prime}+\phi(x) y^{\prime}+\alpha(x) y=f(x)
$$

Theorem 6.1. For the differential equation

$$
y^{\prime \prime}+\phi(x) y^{\prime}+\alpha(x) y=f(x)
$$

The solution is of the form

$$
y=e^{-\int P(x) \mathrm{d} x} \int e^{\int(2 P(x)-\phi(x)) \mathrm{d} x}\left(\int f(x) e^{\int(\phi(x)-P(x)) \mathrm{d} x} \mathrm{~d} x\right) \mathrm{d} x
$$

where, $P(x)$ satisfies the Riccatti Differential Equation:

$$
P^{\prime}(x)=P(x)^{2}-\phi(x) P(x)+\alpha(x)
$$

Proof. Let $P(x)$ and $Q(x)$ be two functions in $x$.

Consider the second-order differential equation in $y$ :

$$
y^{\prime \prime}+[P(x)+Q(x)] y^{\prime}+\left[P^{\prime}(x)+P(x) Q(x)\right] y=f(x)
$$

On re-grouping terms, we have:

$$
\frac{\mathrm{d}\left(y^{\prime}+P(x) y\right)}{\mathrm{d} x}+Q(x)\left[y^{\prime}+P(x) y\right]=f(x)
$$

Given $P(x)$ and $Q(x)$, we can arrive at a solution for $y$ from this equation. Also, we can find and assign $P(x)$ and $Q(x)$ accordingly so that this equation is equivalent to the original form of the given differential equation. Thus,

for $P(x)$ and $Q(x)$, the following must hold:

$$
P(x)+Q(x)=\phi(x)
$$

and,

$$
P^{\prime}(x)+P(x) Q(x)=\alpha(x)
$$


On eliminating $Q(x)$, we get the following Riccatti Equation in $P(x)$ :

$$
P^{\prime}(x)=P(x)^{2}-\phi(x) P(x)+\alpha(x)
$$

Now that $P(x)$ and $Q(x)$ satisfy the needed equations, the solution to the equation can be found:

$$
\frac{\mathrm{d}\left(y^{\prime}+P(x) y\right)}{\mathrm{d} x}+Q(x)\left[y^{\prime}+P(x) y\right]=f(x)
$$

Multiply both sides of the equation by $e^{\int Q(x) \mathrm{d} x}$ ['Integrating Factor']:

$$
e^{\int Q(x) \mathrm{d} x} \frac{\mathrm{d}\left(y^{\prime}+P(x) y\right)}{\mathrm{d} x}+e^{\int Q(x) \mathrm{d} x} Q(x)\left[y^{\prime}+P(x) y\right]=e^{\int Q(x) \mathrm{d} x} f(x)
$$

The left-hand side of this equation is the derivative of " $\left.e^{\int Q(x) \mathrm{d} x}\right)\left[y^{\prime}+P(x) y\right]$ " with respect to $x$.

$$
\mathrm{d}\left(e^{\int Q(x) \mathrm{d} x}\left[y^{\prime}+P(x) y\right]\right)=e^{\int Q(x) \mathrm{d} x} f(x) \mathrm{d} x
$$

On integrating both sides of the equation with respect to $x$ and re-arranging terms, we have:

$$
y^{\prime}+P(x) y=e^{-\int Q(x) \mathrm{d} x} \int f(x) e^{\int Q(x) \mathrm{d} x} \mathrm{~d} x
$$

Multiply both sides of the equation by $e^{\int P(x) \mathrm{d} x}$ ['Integrating Factor']:

$$
e^{\int P(x) \mathrm{d} x} y^{\prime}+e^{\int P(x) \mathrm{d} x} P(x) y=e^{\int(P(x)-Q(x)) \mathrm{d} x} \int f(x) e^{\int Q(x) \mathrm{d} x} \mathrm{~d} x
$$

The left-hand side of this equation is the derivative of $y e^{\int P(x)} \mathrm{d} x$ with respect to $x$.

$$
\mathrm{d}\left(y e^{\int P(x) \mathrm{d} x}\right)=e^{\int(P(x)-Q(x)) \mathrm{d} x}\left(\int f(x) e^{\int Q(x) \mathrm{d} x} \mathrm{~d} x\right) \mathrm{d} x
$$

On integrating both sides of the equation with respect to $x$ and re-arranging terms, we have:

$$
y=e^{-\int P(x) \mathrm{d} x} \int e^{\int(P(x)-Q(x)) \mathrm{d} x}\left(\int f(x) e^{\int Q(x) \mathrm{d} x} \mathrm{~d} x\right) \mathrm{d} x
$$

Also,

$$
Q(x)=\phi(x)-P(x)
$$

Thus,

$$
y=e^{-\int P(x) \mathrm{d} x} \int e^{\int(2 P(x)-\phi(x)) \mathrm{d} x}\left(\int f(x) e^{\int(\phi(x)-P(x)) \mathrm{d} x} \mathrm{~d} x\right) \mathrm{d} x
$$

is the solution. This completes the proof.

\subsection{Closed-Form solutions to certain types of second order linear non-homogeneous non-constant coefficient differential equations}

Based on choices of $\phi(x)$ and $\alpha(x)$, we consider a few types of second-order linear non-homogeneous non-constant coefficient differential equations. The scope of the method is not restricted to the types mentioned below; any equation of the type can be solved as long as we can determine a $P(x)$ i.e. as long as we know any one (particular) solution to the associated Riccati differential equation. Solutions to certain special cases of the Riccati differential equation have been obtained in the existing literature (see [1] ,[3]). We use these solutions to obtain closed-form solutions to certain types of second order linear non-homogeneous non-constant coefficient differential equations. We must be careful to realise that all cases where the exponent is raised to an integral, have arisen from multiplication by the 'Integrating Factor' and hence do not require us to add a constant of integration. 
6.1.1. $y^{\prime \prime}+\phi(x) y^{\prime}+\left(a \phi(x)-a^{2}\right) y=f(x)$.

Here $\alpha(x)=-a^{2}+a \phi(x)$.

The associated Riccati equation is $P^{\prime}(x)=P(x)^{2}-\phi(x) P(x)-a^{2}+a \phi(x)$.

$$
P(x)=a
$$

satisifies this Riccati differential equation. Thus, the solution is:

$$
y=e^{-a x} \int e^{2 a x-\int \phi(x) \mathrm{d} x}\left(\int f(x) e^{-a x+\int \phi(x) \mathrm{d} x} \mathrm{~d} x\right) \mathrm{d} x
$$

6.1.2. $y^{\prime \prime}+\phi(x) y^{\prime}-\frac{\phi(x)}{x} y=f(x)$.

Here $\alpha(x)=-\frac{\phi(x)}{x}$.

The associated Riccati equation is $P^{\prime}(x)=P(x)^{2}-\phi(x) P(x)-\frac{\phi(x)}{x}$.

$$
P(x)=-\frac{1}{x}
$$

satisifies this Riccati differential equation. Thus, the solution is:

$$
y=x \int \frac{e^{-\int \phi(x) \mathrm{d} x}}{x^{2}}\left(\int x f(x) e^{\int \phi(x) \mathrm{d} x} \mathrm{~d} x\right) \mathrm{d} x
$$

6.1.3. $y^{\prime \prime}+\left(a n x^{n-1}-a^{2} x^{2 n}\right) y=f(x)$.

Here $\alpha(x)=a n x^{n-1}-a^{2} x^{2 n}$ and $\phi(x)=0$.

The associated Riccati equation is $P^{\prime}(x)=P(x)^{2}+a n x^{n-1}-a^{2} x^{2 n}$.

$$
P(x)=a x^{n}
$$

satisifies this Riccati differential equation. Thus, the solution is:

$$
y=e^{\beta(x)} \int e^{-2 \beta(x)}\left(\int f(x) e^{\beta(x)} \mathrm{d} x\right) \mathrm{d} x
$$

where,

$$
\beta(x)=-\frac{a x^{n+1}}{n+1} .
$$

6.1.4. $y^{\prime \prime}+\phi(x) y^{\prime}+\left(a n x^{n-1}-a^{2} x^{2 n}+a x^{n} \phi(x)\right) y=f(x)$.

Here $\alpha(x)=a n x^{n-1}-a^{2} x^{2 n}+a x^{n} \phi(x)$.

The associated Riccati equation is $P^{\prime}(x)=P(x)^{2}-\phi(x) P(x)+a n x^{n-1}-$ $a^{2} x^{2 n}+a x^{n} \phi(x)$.

$$
P(x)=a x^{n}
$$

satisifies this Riccati differential equation. Thus, the solution is:

$$
y=e^{\beta(x)} \int e^{-\left(2 \beta(x)+\int \phi(x) \mathrm{d} x\right)}\left(\int f(x) e^{\beta(x)+\int \phi(x)} \mathrm{d} x\right) \mathrm{d} x
$$

where,

$$
\beta(x)=-\frac{a x^{n+1}}{n+1} .
$$


6.1.5. $y^{\prime \prime}+\left(R^{\prime}(x)-R(x)^{2}\right) y=f(x)$.

Here $\alpha(x)=R^{\prime}(x)-R(x)^{2}$ and $\phi(x)=0$.

The associated Riccati equation is $P^{\prime}(x)=P(x)^{2}+R^{\prime}(x)-R(x)^{2}$.

$$
P(x)=R(x)
$$

satisifies this Riccati differential equation. Thus, the solution is:

$$
y=e^{-\int R(x) \mathrm{d} x} \int e^{\int 2 R(x) \mathrm{d} x}\left(\int f(x) e^{-\int R(x) \mathrm{d} x} \mathrm{~d} x\right) \mathrm{d} x
$$

\section{Application to recurrence relations}

In this section, we apply a method similar to methods used in previous sections to obtain a closed-form solution to recurrence relations. In general, most recurrence relations can be solved using methods similar to those described above for their continuous counterparts, differential equations.

We first obtain a closed-form solution for recurrence relations of the first order and then apply it towards obtaining a solution to linear non-homogeneous constant-coefficient recurrence relations.

Theorem 7.1. For the recurrence relation,

$$
x_{n}=\alpha x_{n-1}+f(n)
$$

the solution is

$$
x_{n}=a^{r} x_{n-r}+\sum_{p=0}^{r-1} a^{p} f(n-p)
$$

for any $r \in N$ such that $1 \leq r \leq n-1$.

Proof. The result is trivial. We have,

$x_{n}=a x_{n-1}+f(n)$

For $n-1$, we have

$x_{n-1}=a x_{n-2}+f(n-1)$

Muliplying both sides of this equation by $a$, we have

$$
a x_{n-1}=a^{2} x_{n-2}+a f(n-1)
$$

Similarly for $n-2$, we have

$$
x_{n-2}=a x_{n-3}+f(n-2)
$$

Muliplying both sides of this equation by $a^{2}$, we have

$a^{2} x_{n-1}=a^{3} x_{n-2}+a^{2} f(n-1)$

In general, for $n-k$, where, $k \in N$ such that $1 \leq k \leq r$, we have

$a^{k} x_{n-k}=a^{k+1} x_{n-k-1}+a^{p} f(n-k)$

On adding equations of the form of (4) for $k=1,2 \ldots r$, we have

$$
x_{n}=a^{r} x_{n-r}+\sum_{p=0}^{r-1} a^{p} f(n-p)
$$

This completes the proof. Now, we proceed onto second order relations. 
Theorem. For the recurrence relation,

$$
x_{n}+\alpha x_{n-1}+\beta x_{n-2}=f(n)
$$

where $n \geq 2, n \in N$ and $\alpha$ and $\beta$ are constants such that $\beta \neq 0$,

the solution is:

for $\alpha^{2} \neq 4 \beta$ i.e. $a \neq b$,

$$
x_{n}=a^{n-1} x_{1}+\frac{b\left(x_{1}-a x_{0}\right)\left(a^{n-1}-b^{n-1}\right)}{a-b}+a^{n} \sum_{t=2}^{n}\left(\frac{b}{a}\right)^{t}\left[\sum_{p=2}^{t} b^{-p} f(p)\right]
$$

for $\alpha^{2}=4 \beta$ i.e. $a=b$,

$$
x_{n}=n a^{n-1} x_{1}-(n-1) a^{n} x_{0}+a^{n} \sum_{t=2}^{n}\left[\sum_{p=2}^{t} a^{-p} f(p)\right]
$$

where, $a$ and $b$ are roots of the quadratic equation :

$$
t^{2}+\alpha t+\beta=0
$$

Proof. Since $a$ and $b$ are roots of the quadratic equation $t^{2}+\alpha t+\beta=0$,

$$
\begin{gathered}
\alpha=-a-b \\
\beta=a b .
\end{gathered}
$$

On substituting $\alpha$ and $\beta$ into the recurrence relation, we have

$$
x_{n}-a x_{n-1}-b x_{n-1}+a b x_{n-2}=f(n)
$$

On re-arranging the terms, we have

$$
\left(x_{n}-a x_{n-1}\right)-b\left(x_{n-1}-a x_{n-2}\right)=f(n)
$$

Let $z_{n}=x_{n}-a x_{n-1}$. Thus,

$$
z_{n-1}=x_{n-1}-a x_{n-2} .
$$

On substituting $z_{n}$ and $z_{n-1}$ into the recurrence relation, we have

$$
z_{n}=b z_{n-1}+f(n)
$$

Applying Theorem 7.1 when $r=n-1$, we have

$$
z_{n}=b^{n-1} z_{1}+\sum_{p=0}^{n-2} b^{p} f(n-p)
$$

With a change of variables by replacing $p$ with $n-p$, we have

$$
z_{n}=b^{n-1} z_{1}+b^{n} \sum_{p=2}^{n} b^{-p} f(p)
$$

Let

$$
g(n)=b^{n-1} z_{1}+b^{n} \sum_{p=2}^{n} b^{-p} f(p)
$$

Since $z_{n}=x_{n}-a x_{n-1}$, we have

$$
\begin{aligned}
& x_{n}=a x_{n-1}=g(n) \\
& x_{n}=a x_{n-1}+g(n)
\end{aligned}
$$


Applying Theorem 7.1 when $r=n-1$, we have

$$
x_{n}=a^{n-1} x_{1}+\sum_{q=0}^{n-2} a^{q} g(n-q)
$$

Substituting for $g(n)$, we have

$$
x_{n}=a^{n-1} x_{1}+\sum_{q=0}^{n-2} a^{q}\left[b^{n-q-1} z_{1}+b^{n-q} \sum_{p=2}^{n} b^{-p} f(p)\right]
$$

On setting $t=n-q$, we have

$$
\begin{gathered}
x_{n}=a^{n-1} x_{1}+\sum_{t=2}^{n} a^{n-t}\left[b^{t-1} z_{1}+b^{t} \sum_{p=2}^{n} b^{-p} f(p)\right] \\
x_{n}=a^{n-1} x_{1}+\sum_{t=2}^{n} a^{n-t} b^{t-1} z_{1}+a^{n} \sum_{t=2}^{n}\left(\frac{b}{a}\right)^{t}\left[\sum_{p=2}^{t} b^{-p} f(p)\right]
\end{gathered}
$$

From the definition of $z, z_{1}=x_{1}-a x_{0}$.

If $a \neq b$, on re-arranging the terms and applying the formula for the sum of a geometric series, we have

$$
x_{n}=a^{n-1} x_{1}+\frac{b\left(x_{1}-a x_{0}\right)\left(a^{n-1}-b^{n-1}\right)}{a-b}+a^{n} \sum_{t=2}^{n}\left(\frac{b}{a}\right)^{t}\left[\sum_{p=2}^{t} b^{-p} f(p)\right]
$$

Or else if $a=b$, we have

$$
\begin{gathered}
x_{n}=a^{n-1} x_{1}+a^{n-1}(n-1)\left(x_{1}-a x_{0}\right)+a^{n} \sum_{t=2}^{n}\left[\sum_{p=2}^{t} a^{-p} f(p)\right] \\
x_{n}=n a^{n-1} x_{1}-(n-1) a^{n} x_{0}+a^{n} \sum_{t=2}^{n}\left[\sum_{p=2}^{t} a^{-p} f(p)\right]
\end{gathered}
$$

This completes the proof.

\section{Conclusion}

Thus closed form solutions for the second order linear constant-coefficent non-homogeneous differential equation, the third order linear constant-coefficent non-homogeneous differential equation and a more generalised variant of the second order non-homogeneous Cauchy-Euler equation have been derived.In contrast to exisiting methods involving usage of ansatz, this method does not require guessing techniques. The method has then been generalized for the $n^{t h}$ order linear constant-coefficent non-homogeneous differential equation. The problem of solving such an equation is then effecively reduced to solving a system of $n$ algebraic equations, and then evaluating an integral. It can be observed that such an equation of order $n$ will have a solution comprising an integral iterated $n$ times. Along similar lines, the solution to the second order linear non-constant-coefficent non-homogeneous differential equation has been obtained, by associating it with a Riccati differential equation. Then, once we have any one (particular) solution to the Riccati differential equation, we need only calculate an integral to obtain a solution to the second order differential equation. The motivation of the method(s) lies in the effective regrouping of terms to enable reduction of these equations into first order equations, as has been described in detail in each of the sections, particularly in Section 6. The method is then applied to recurrence relations, first by obtaining a trivial result for first order relations and then using the result to derive closed-form solutions to second order relations. 


\section{References}

[1] Andrei Polyanin \& Valentin Zaitsev, Handbook of Exact Solutions for Ordinary Differential Equations, Second Edition, Chapman and Hall/CRC, (2002).

[2] William Boyce \& Richard Di Prima, Elementary Differential Equations, Eighth Edition, Wiley, (2004).

[3] Daniel Zwillinger, Handbook of Differential Equations, Third Edition, Academic Press, (1997). 